\title{
Impact of SARS-CoV-2 Pandemic on Patients with Primary Immunodeficiency
}

\author{
Samaneh Delavari ${ }^{1} \cdot$ Hassan Abolhassani ${ }^{1,2}$ (D) Farhad Abolnezhadian ${ }^{3} \cdot$ Fateme Babaha $^{1,4} \cdot$ Sara Iranparast $^{5}$. \\ Hamid Ahanchian ${ }^{6} \cdot$ Nasrin Moazzen $^{6}$. Mohammad Nabavi ${ }^{7}$. Saba Arshi ${ }^{7}$. Morteza Fallahpour ${ }^{7}$. \\ Mohammad Hassan Bemanian ${ }^{7} \cdot$ Sima Shokri $^{7} \cdot$ Tooba Momen $^{8} \cdot$ Mahnaz Sadeghi-Shabestari $^{9} \cdot$ Rasol Molatefi $^{10}$. \\ Afshin Shirkani ${ }^{11}$. Ahmad Vosughimotlagh ${ }^{12}$. Molood Safarirad ${ }^{12} \cdot$ Meisam Sharifzadeh $^{13} \cdot$ Salar Pashangzadeh $^{1}$. \\ Fereshte Salami ${ }^{1}$ • Paniz Shirmast ${ }^{1}$ - Arezou Rezaei ${ }^{1} \cdot \operatorname{Tannaz}$ Moeini Shad ${ }^{1} \cdot$ Minoo Mohraz $^{14} \cdot$ Nima Rezaei $^{1}$. \\ Lennart Hammarström ${ }^{2} \cdot$ Reza Yazdani $^{1} \cdot$ Asghar Aghamohamamdi $^{1}$
}

Received: 23 August 2020 / Accepted: 16 November 2020 / Published online: 1 December 2020

(C) The Author(s) 2020

\begin{abstract}
Although it is estimated that COVID-19 life-threatening conditions may be diagnosed in less than 1:1000 infected individuals below the age of 50, but the real impact of this pandemic on pediatric patients with different types of primary immunodeficiency (PID) is not elucidated. The current prospective study on a national registry of PID patients showed that with only 1.23 folds higher incidence of infections, these patients present a 10-folds higher mortality rate compared to population mainly in patients with combined immunodeficiency and immune dysregulation. Therefore, further management modalities against COVID-19 should be considered to improve the survival rate in these two PID entities using hematopoietic stem cell transplantation and immunomodulatory agents.
\end{abstract}

Keywords COVID-19 $\cdot$ Primary immunodeficiency $\cdot$ Severe viral infection $\cdot$ Mortality rate

\section{Introduction}

The novel coronavirus disease, known as coronavirus disease 2019 (COVID-19), is an acute infectious respiratory disease that first emerged in Wuhan, China, in late 2019 and is characterized as a pandemic in mid-March 2020 by World Health Organization [1]. It has been found that COVID-19 causes severe acute respiratory syndrome (SARS, therefore coined as SARS-CoV-2) similar to two other RNA viruses from the Coronoviridea family SARS-CoV-1 and Middle East

Samaneh Delavari and Hassan Abolhassani contributed equally to this work.

Article Summary Line Patients with combined immunodeficiency and immune dysregulation are at a high risk of mortality due to SARS-CoV-2 compared to other types of primary immunodeficiency.

Hassan Abolhassani

hassan.abolhassani@ki.se

Asghar Aghamohamamdi

aghamohammadi@tums.ac.ir

Extended author information available on the last page of the article respiratory syndrome coronavirus (MERS-CoV). SARSCoV-2 main route of entry to a human host is the angiotensin-converting enzyme 2 (ACE2) receptor. ACE2 receptor is ubiquitously expressed on the surfaces of various cell types, including cells of the airway epithelium which is the major site of infection [2]. Besides the ACE2 receptor, SARSCoV-2 uses the transmembrane serine protease 2 (TMPRSS2), a cellular serine protease, for the host cell entry, which activates the SARS-CoV-2 spike protein by cleaving the Furin site at the S1/S2 subunits [3, 4]. Upon entry, the virus can subsequently affect endosomes, and eventually, fuse viral and lysosomal membranes [5]. Coronaviruses are pathogens affecting both humans and animals; particularly SARS-CoV-2 is highly contagious and can be transmitted directly through respiratory droplets often even before the infected person shows symptoms or indirectly by touching infected surfaces and following inoculation within mucosal layers $[2,6]$.

SARS-CoV-2 infection results in the development of an unusual form of pneumonia and can cause acute respiratory distress syndrome (ARDS) [7]. More than 35 million infected cases were diagnosed worldwide, with a mortality rate of $4 \%$ 
(1-20\%) and about $20 \%$ of those who get COVID-19 become seriously ill and require oxygen, with $5 \%$ becoming critically ill and needing intensive care (https://www.who.int/ emergencies/diseases/novel-coronavirus-2019/question-andanswers-hub/q-a-detail/coronavirus-disease-covid-19), mainly in old patients with multiple comorbidities. Of note, it is estimated that life-threatening conditions may be observed in less than 1:1000 infected individuals below the age of 50 [7], suggesting some underlying susceptibility factors in this selected group of patients. Hence, it is expected to see severe COVID-19 cases in individuals with primary immunodeficiencies (PIDs). Due to poor cellular immunity and viral control, disease severity is expected to be higher in combined immunodeficiencies than patients with humoral defects. Patients with immune dysregulation may also be vulnerable to the disease because of the risk of an adverse unregulated inflammatory response in these patients. Noteworthy, patients with defects in their innate immune system, especially those with defects in the interferon (IFN) pathway, seem to face more frequently a critical course of the disease [8]. PID prevalence (range 1:8500-1:100000 for symptomatic patients) and the proportion of PID entities (more than 400 heterogeneous defects) in each country may impact the number of patients at risk due to COVID-19. Recent discoveries identified the role of defective immune-related genes involved in RNA-virus infection susceptibilities with a critical function in IFN pathways including IFN receptors (IFNAR1 and IFNAR2), signal transducer and activator of transcription of IFN (STAT1 and $S T A T 2$ ), IFN regulatory factors (IRF7, IRF9, and IFIH1), and RNA polymerase III (POLR3A, POLR3C, and POLR3F) [9].

To investigate this hypothesis, we conducted a prospective study to compare the rate and outcomes of COVID-19 infection between diagnosed cases in the Iranian PID registry with population-based data.

\section{Methods}

\section{Iranian Primary Immunodeficiency Registry (IPIDR)}

This study was conducted as a cohort of patients, prospectively enrolled in the IPIDR from the "National PID Network" [10]. The IPIDR is managed by the Research Center for Immunodeficiencies (Tehran, Iran) and its main aim is to provide epidemiological, clinical, and molecular data of PID in Iran. By the latest estimation in 2020, Iran has a population of $84,012,442$ citizens (with an average annual birth rate of $1,300,000$ ) and according to the age structure, $23.7 \%$ are less than 14 years old. Data on COVID-19 infection in PID patients were compared with the normal population $(471,772$ patients, incidence of 2036 cases per day, 26,957 total death, https://www.worldometers.info/coronavirus/, reported from Iranian Ministry of Health, Tehran, Iran, access data 4
October 2020). This study received approval from the Ethics Committee of the Tehran University of Medical Science. Moreover, written informed consent has been obtained from all patients, their parents, or legal guardians.

The registry database is hosted in the Children's Medical Center (Tehran, Iran) which serves as a referral hospital for suspected or diagnosed PID cases. Moreover, 38 medical centers, affiliated to 27 medical science universities, collaborated in the registry program from the major provinces of the country to form the PID network. All participating centers had access to national guidelines [11] and necessary laboratory equipment for clinical and immunological evaluations [10]. Subsequently, cases with a suspected diagnosis were referred and re-evaluated in the Children's Medical Center for a definitive diagnosis.

\section{Clinical and Immunologic Diagnoses in COVID-19- Infected PID Patients}

The clinical diagnosis of the PID patients was made according to the criteria of the European Society for immunodeficiencies (ESID) [12]. A questionnaire surveyed the patients' demographic information, age of disease onset, age of diagnosis, family history, a detailed clinical history that included vaccination history and associated adverse reactions, recurrent infections, physical examination findings, laboratory data, and treatment history. Secondary defects of the immune system, including those caused by human immunodeficiency virus (HIV) were ruled out. Laboratory evaluations were performed in the study group as indicated, including complete blood and differential counts, serum protein profile and immunoglobulin levels, serum IgG subclass levels, isohemagglutinin titers, specific antibody responses, disease-specific autoantibody measurements, flow cytometric evaluation of lymphocyte subsets, nitro blue tetrazolium dye/dihydrorhodamine test, granulocyte function and chemotaxis tests, lymphocyte transformation and $\mathrm{T}$ cell function tests, radiosensitivity, measurement of complement component levels, and hemolytic complement activity [10]. Microbiological, pathological and imaging evaluations were performed for clinical diagnosis when required. A computerized database program (new registry section in http://rcid.tums.ac.ir/, access data 4 October 2020) was implemented for data entry. After reviewing the cases by the administrator of the system for duplicated cases, patients with incomplete diagnostic criteria were excluded. The online database was updated frequently for approved patients and all follow-up data sent by the end of the study period were included. If the diagnosis of PID were confirmed before the time of COVID-19 patients, following evaluation were performed including new clinical presentation, high-resolution computed tomography (HRCT), and reverse transcriptasepolymerase chain reactions (RT-PCR) as well as complete blood count (CBC), C-reactive protein (CRP), and erythrocyte 
sedimentation rate (ESR). For patients with a recent diagnosis of PID, a comprehensive questionnaire was provided and filled out for each patient which consists of demographical data, clinical manifestations related to their PID and COVID-19, laboratory tests findings such covering both PID and COVID-19 diagnoses confirmation. Furthermore, the type of treatment and outcome were also provided for each patient.

\section{Genetic Analysis and Diagnoses in COVID-19-Infected PID Patients}

Genomic DNA was extracted from whole blood from patients who agreed with genetic tests and for patients with classical clinical presentations suggestive of a specific PID, targeted sequencing was performed (Table S1). For patients in whom targeted sequencing failed or who had a clinical presentation resembling several genetic defects, whole-exome sequencing was performed to detect single nucleotide variants, insertion/ deletions, and large deletions using a pipeline described previously $[13,14]$. Candidate variants were evaluated by the Combined Annotation Dependent Depletion (CADD) algorithm and an individual gene cutoff given by using the Mutation Significance Cutoff (MSC) was considered for impact predictions [15]. The Gene Damage Index (GDI) server and the Human Gene Connectome (HGC) were used to making a combined effect prediction [15]. The pathogenicity of all disease attributable gene variants was re-evaluated using the updated guideline for interpretation of molecular sequencing by the American College of Medical Genetics and Genomics criteria $[16,17]$, considering the allele frequency in the population database, computational data, immunological data, familial segregation and parental data (confirmatory Sanger sequencing for probands and their parents), and clinical phenotyping.

\section{Classification of COVID-19-Infected PID Patients}

After confirmation of their clinical and genetic diagnosis, patients were classified according to the International Union of Immunological Societies (IUIS) updated classification including 9 categories of immunodeficiencies affecting cellular and humoral immunity (non-syndromic combined immunodeficiency or CID), combined immunodeficiencies with associated or syndromic features (syndromic CID), predominantly antibody deficiencies (PAD), diseases of immune dysregulation, congenital defects of phagocyte number or function (phagocytic disorders), autoinflammatory disorders, defects in intrinsic and innate immunity, complement deficiencies, and phenocopies of inborn errors of immunity [9].

\section{Results}

Among 4718 registered patients, 2754 patients (998 females, median age 108 months) were alive and on monthly follow-up (58.3\%, before the emergence of COVID-19 first report in the country 19 February 2020). During this period, each patient had on average 8 follow-up visits in our peripheral centers, and the COVID-19 test was performed in patients with the clinical triad of cough, fever, and dyspnea. To date, 19 patients ( 7 females, median age 106 months) were confirmed with positive reverse transcriptase-polymerase chain reactions (RT-PCR) SARS-CoV-2 test (1:144 incidence compared to $1: 178$ in the total population, 1.23 folds higher risk of infections). It seems that this measurement is underestimated since PID patients/families were trained for tight isolation compared to other immunocompetent individuals in the population. Exposure to SARS-CoV-2 from an unknown source or a source outside the child's family accounted for $84.2 \%$ of the cases of infection (15.8\% of PID patients have a medical history of exposure to another COVID-19-infected family members prior to their hospital admission). Detailed comparison of infection rates between PID patients versus the total population based on adjusted age-groups is shown in Fig. S1.

Combined immunodeficiencies ( $n=10$, all without hematopoietic stem cell transplantation or HSCT, 47.0\%) were the major PID entity among COVID-19 positive cases followed by humoral immunodeficiencies $(n=4)$, phagocytic defects $(n=2)$, immune dysregulation $(n=2)$, and autoinflammatory disorders ( $n=1$, Table 1$)$. Of note, no COVID-19 infection was observed in patients with innate or complement deficiencies (117 and 85 total registered alive patients, respectively). COVID-19 infections alone or in complex with other manifestations were the first clinical presentation of PID in 4 patients, mainly combined immunodeficiencies (Tables 2 and 3 ). Current genetic data on the evaluated patients is indicated in Fig. S2. Of note, COVID-19 infection was not yet reported in any of the alive patients with a defect in the IFN pathway $(n=$ 23, data not shown). The geographical distribution of identified patients matched with the cumulative incidence of PID, but not with the incidence of COVID-19 in the general population (Fig. S3).

Details of clinical manifestations and laboratory findings of patients before and after SARS-CoV-2 infections are summarized in Tables 2 and 3, S2 and S3. The majority of patients had a history of lower respiratory tract infection before COVID-19 (89.4\%, except for two patients with STK4 and IL1RN deficiencies). Severe distress requiring respiratory support was documented as COVID-19 features in 10 patients from which 7 had lymphoproliferation (70\%) including lymphadenopathy, hepatosplenomegaly, and non-necrotizing granulomatous inflammation. Moreover, bronchiectasis $(21.0 \%)$, cardiovascular complications $(10.5 \%)$, and liver failure $(10.5 \%)$ were observed in patients with poor prognosis. Of 


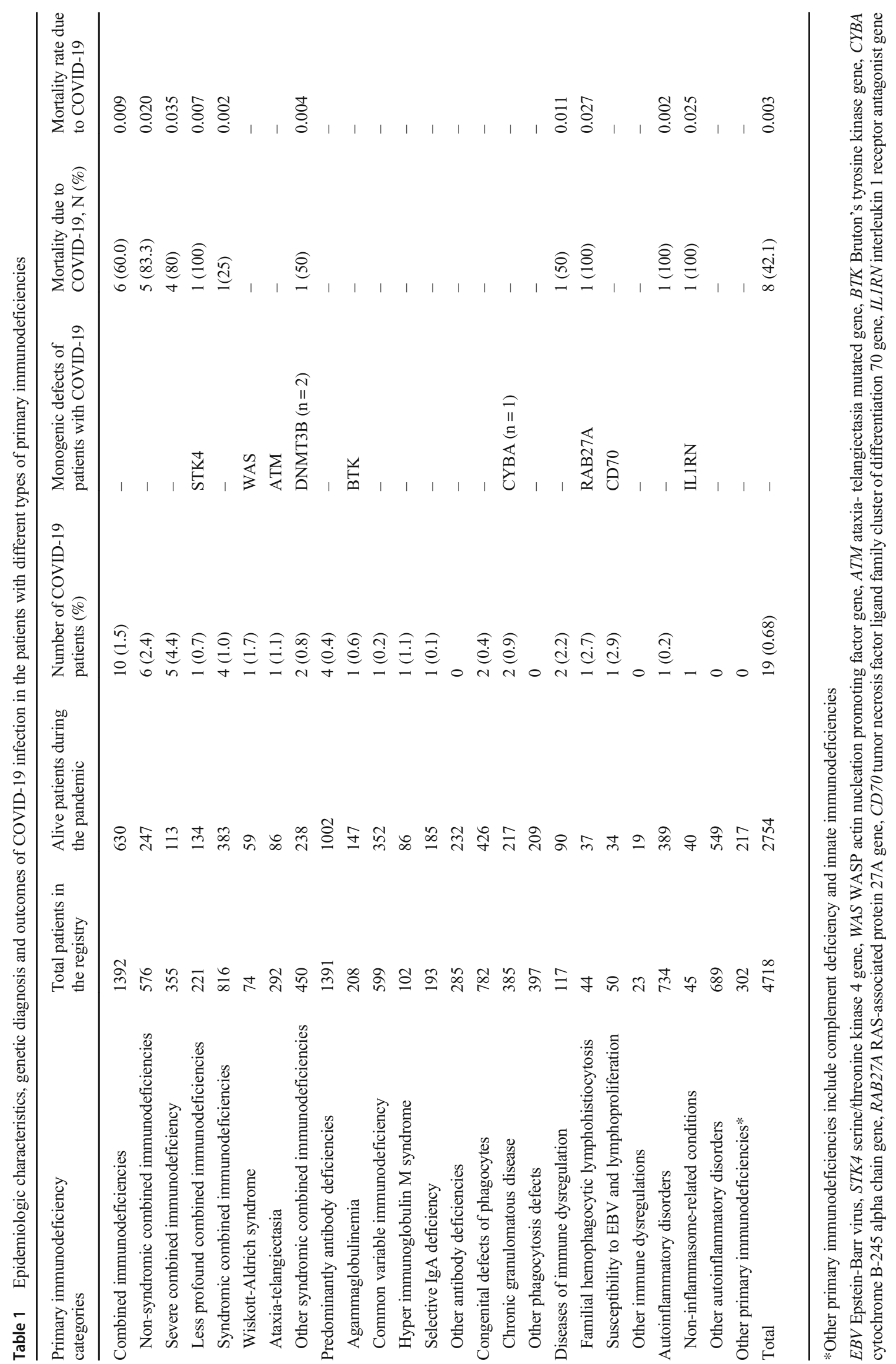




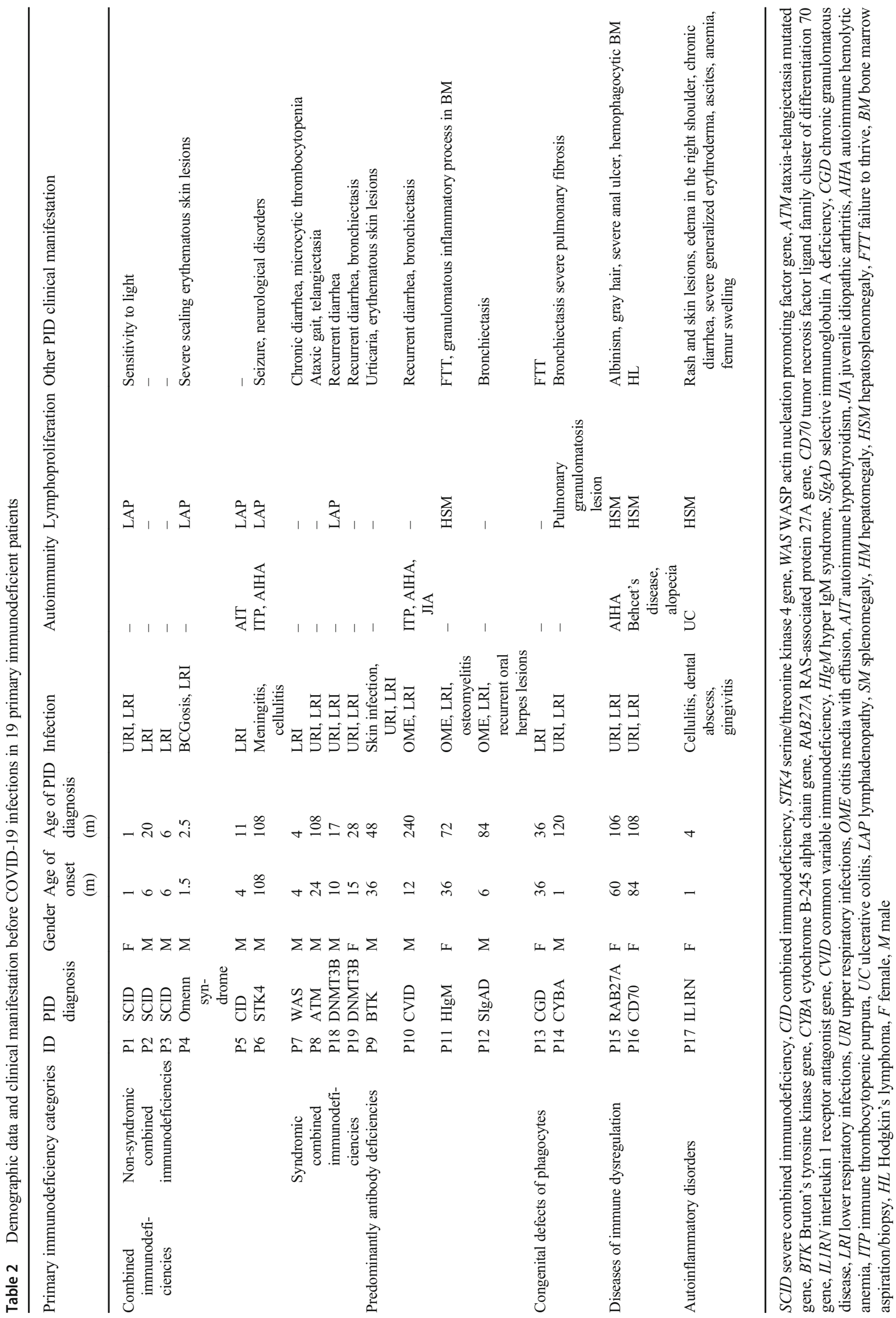




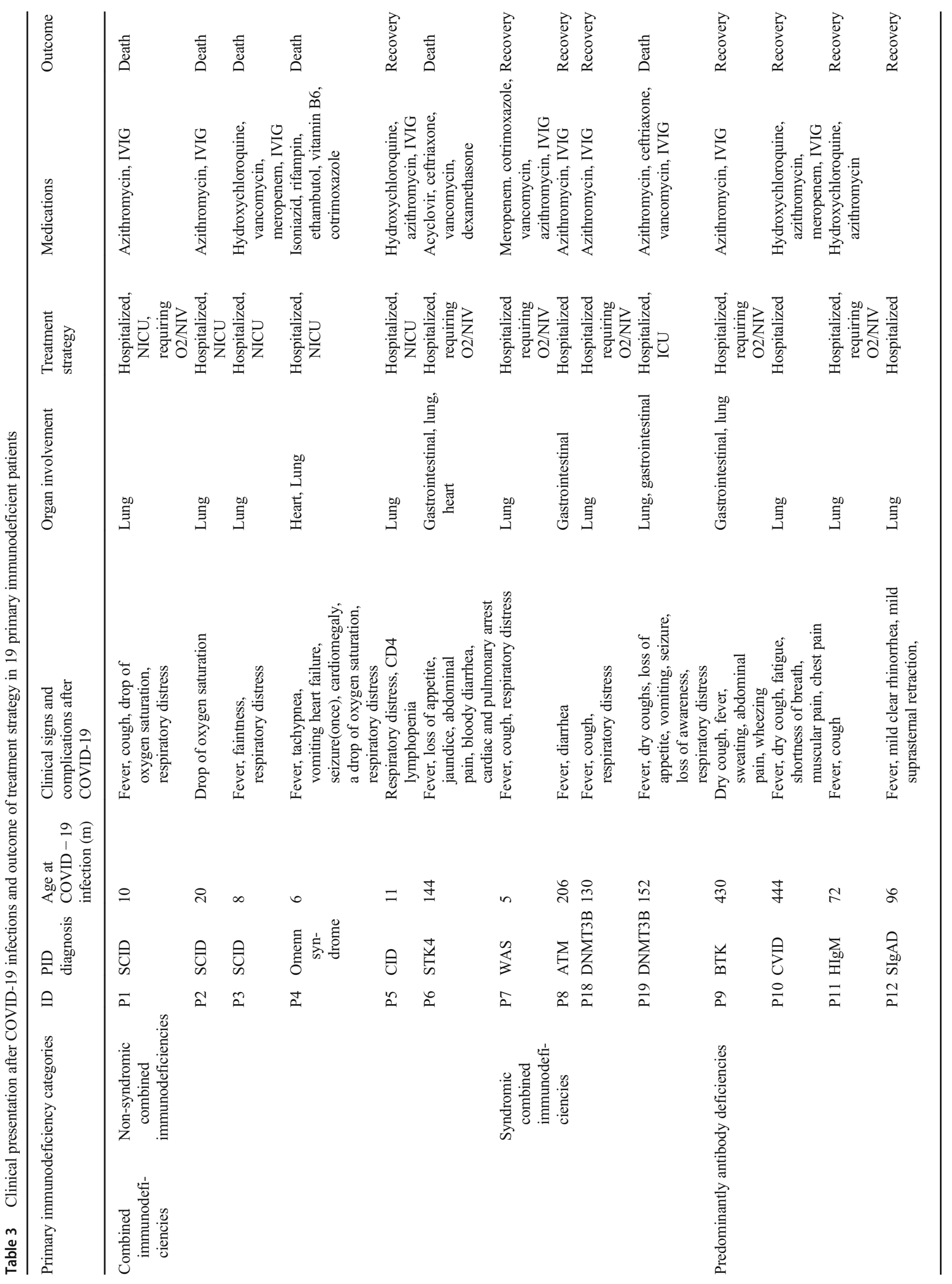




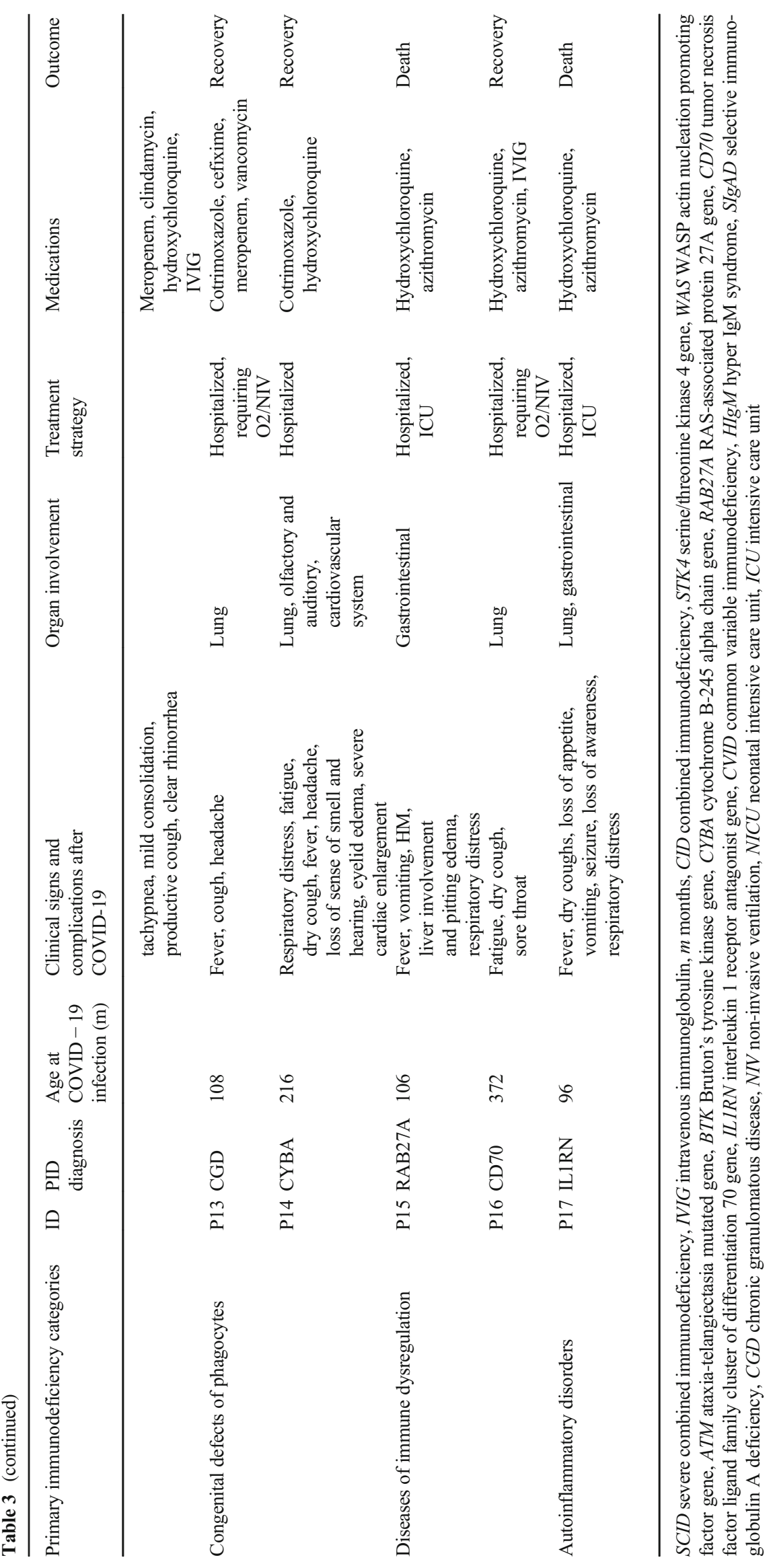


note, bone marrow analysis and laboratory data of COVID19-infected patients with immune dysregulation (P15 and P16) did not reveal hemophagocytic lymphohistiocytosis (HLH) activity at the time of the current study. Acute-phase reactant proteins were negative in 8 patients, particularly in patients with severe combined immunodeficiency (SCID) and phagocytosis defects. Imaging findings on COVID-19 pulmonary complications of PID patients varied from mild prominence of bronchovascular markings to mucus plugging, prebronchial thickening, diffuse patchy opacities, collapse/ consolidations, mosaic perfusion, and ground glass interstitial disease, mirroring of the severity of diagnosed PID (Figs. S4 S15).

Among the identified PID cases with COVID-19, 8 patients deceased $(42.1 \%$, Table 1$)$ indicating a 10 -folds higher mortality rate in PID patients compared to the population (0.003 vs. $0.0003, p<0.001)$. Of note, the most lethal COVID-19 infection among PID entities was observed in patients with SCID (0.03) and familial hemophagocytic lymphohistiocytosis (FHL, 0.027). This notion confirms the previous hypothesis that SARS-CoV-2 are more lifethreatening among patient with cellular immunodeficiency and immune dysregulation with almost 150 -folds higher risk of mortality. All infected patients with less profound combined immunodeficiencies, FHL, and autoinflammatory disorders succumbed to COVID-infection. Monthly intravenous immunoglobulin replacement therapy was continued during the COVID-19 infection period in 12 patients, of which $66.6 \%$ recovered from the infection (4 deceased patients had combined immunodeficiencies, Table 2).

\section{Discussion}

The current knowledge about COVID-19 in cases with underlying immunodeficiency is scarce [18-24]. Comparing to previous data reported mainly primary antibody deficient and male patients with COVID-19 infection (Table S4), we did not observe the dominancy of infected patients in this PID category despite their high frequency among the national registry with no deceased patient. In the first observation in 7 cases from Italy, all were antibody-deficient patients with $14.2 \%$ mortality [25]. In another report from 582 children and adolescents in Europe with PCR-confirmed SARS-CoV2 infection, 3 patients ( $0.5 \%$ of total cases) had a previously diagnosed PID comprising common variable immunodeficiency, congenital neutropenia, and Schimke immunoosseous dysplasia [26].

These data resembling the findings of the recent study by Parri et al. [27] where they highlighted immunological complications may underlie $7.4-12.5 \%$ of COVID-19 patients reported from pediatric emergency departments. However, our study may strengthen the current understanding of the impact of the pandemic on all subgroups of PID and highlights the requirement of tight measurements in children affected mainly with combined immunodeficiency and immune dysregulation. Of note, several recent studies identified that lymphopenia with prominent decreased $\mathrm{T}$ cell (mainly $\mathrm{CD} 8^{+}$cytotoxic $\mathrm{T}$ cell) counts are associated with severe COVID-19 condition and mortality [3, 28-31]. Indeed, lymphopenia seems to correlate with the cytokine profile of the severe patients which resemble our observation in PID patients [30, 32]. On the other hand, the recent multinational cohort reported by ESID (94 patients, 37\% were with mild symptoms or asymptomatic, $9.5 \%$ mortality) revealed that older patients with combined immunodeficiency (14 patients in this cohort all recovered, Table S4) manifested milder presentations compared to our patients, which may reflect different underlying diseases, but also access to optimal treatment mainly HSCT [33].

While the induction of effective cellular immunity is likely essential for the COVID-19 control, dysregulated T cell activation (by overproduction of IFN associated cytokines promoting retention of lymphocytes in lymphoid organs) may underly main immunopathology and contribute to disease severity in COVID-19 patients [32, 34-36]. Although the exposure to pathogens may be lower in PID patients due to more strict self-isolation, it should be noted that the majority of PID cases require to visit the hospital and medical centers regularly which may predispose them even more to exposure to different pathogens as well as COVID-19. Even though our approach between PID patients and the general population were similarly based on decided national COVID-19 protocols (RT-PCR test was performed in cases with a suggestive triad of fever, cough, and dyspnea), it should be declared that some infected cases from the population may be missed with this inclusion criteria. Indeed, PID patients are followed-up more regularly, whereas the infected immunocompetent individual with mild infections in the normal population may not refer to the medical centers. Therefore, future epidemiological studies in other countries are required to evaluate this notion.

Our current genetic findings suggest a higher mortality rate on special molecular defects associated with deficiency of the IL-1 receptor (DIRA deficiency), STK4 deficiency (combined immunodeficiency), and RAB27A deficiency (diseases of immune dysregulation) with COVID-19. However, this data need to be supported with future evidence from other PID cohorts worldwide since these disorders are extremely rare. The notion observed could alert physicians on whether the usage of IL-1 inhibitors, such as anakinra, might be helpful for the treatment of COVID-19, and this decision should be made individually for each patient based on their genetic diagnosis and medical condition [37]. Although admission to IL-1 inhibitor in intensive care unit admitted cases could significantly reduce the mortality rate (hazard ratio of $0.22 \mathrm{com}$ pared to non-receivers), this might only be indicated in patients with hemophagocytic lymphohistiocytosis, macrophage 
activation syndrome with certain overactivation of inflammasome pathway. Of note, recent genetic analysis of 4 young brother pairs (range, 21-32 years) with respiratory insufficiency due to severe COVID-19 suggested TLR7 deficiency with reduced production of IFN- $\gamma$, a type II IFN as the underlying cause [38]. Moreover, review literature [25, 33, 38, $39,40,41]$ on PID patients with COVID-19 infections might also indicate a possible association of the disease with mutations in BTK (9 patients), IRF7 (7 patients) and TLR4 (4 patients), genes (Table S4). Although we did not identify COVID-19 infection in any of our patients with innate immune defects, this observation may also indicate future follow-up on patients with defects in TLR and IFN pathways are required to understand the genetic predisposition and pathogenesis of COVID-19 correctly, as 23 patients with diseasecausing variants and life-threatening COVID-19 have been reported recently [39].

Our findings showed that about $30 \%$ of the total Iranian population is younger than 20 years and indeed it has been evident that they presented a very low frequency of COVID19 infections and almost no mortality compared to other age categories (Fig. S1). Whereas about 57\% of PID patients are under the age of 20, and a significant number of COVID-19infected patients were observed in this age group, and the majority of them died which indicates an obvious contrast to the population. Moreover, in the total population, $70 \%$ are over 20 years old comprising a considerable percentage of patients with SARS-CoV-2 infection and the highest COVID-19 mortality. In contrast, although about $43 \%$ of PID patients are older than 20 years (1184 patients), only three SARS-CoV-2 positive patients were recorded and none of them had died due to SARS-CoV-2 which is again markedly different from the normal population. We actually observed a 10 -fold higher mortality rate and a reverse pattern of the agestructure in COVID-19-infected PID patients compared to the population. This is also indeed higher than the mortality rate documented in children and adolescents in Europe where only 4 patients died, all older than 10 years, with a crude fatality rate of 0.0069 . Of note, one of those deceased patients (25\%) had underlying immunodeficiency due to HSCT [26]. But it is important to highlight that recessive forms of PID might play a role in the difference observed between our PID study and the European pediatric cohort since these severe PIDs may possibly increase the incidence of more severe COVID19 cases during childhood.

\section{Conclusion}

Although COVID-19 is generally a mild disease in children and adolescents (due to the low ACE2 receptor expression and functional adaptive immunity) [42, 43], a fraction of them including PID patients may develop severe disease requiring intensive unit care admission and even fatal outcome. Future studies may corroborate the individual risk of different PID disorders and clarify the potential need for preemptive measures for specific subsets of PID patients at high risk of a critical course of COVID19.

Supplementary Information The online version contains supplementary material available at https://doi.org/10.1007/s10875-020-00928-x.

\section{Authors' Contributions}

(1)The conception and design of the study.

(2) Acquisition of data.

(3) Analysis and interpretation of data.

(4) Drafting the article.

(5) Revising it critically for important intellectual content.

(6) Final approval of the version to be submitted.

(7) Agree to be accountable for all aspects of the work in ensuring that questions related to the accuracy or integrity of any part of the work are appropriately investigated and resolved.

SD, HA $(2,3,4,6,7)$, and FA, FB, SI, HA, NM, MB, SA, MF, MHB, SS, TM, MSS, RM, AS, AV, MS, MSh, SP, FS, PS, AR, TMS $(2,5,6,7)$ MM, NR, LH, RY $(2,3,5,6,7)$ AA $(1,3,4,5,6)$. All authors read and approved the final manuscript.

Funding Open access funding provided by Karolinska Institute.

\section{Compliance with Ethical Standards}

Conflict of Interest The authors have no relevant affiliations or financial involvement with any organization or entity with a financial interest in or financial conflict with the subject matter or materials discussed in the manuscript. This includes employment, consultancies, honoraria, stock ownership or options, expert testimony, grants, or patents received or pending or royalties.

Open Access This article is licensed under a Creative Commons Attribution 4.0 International License, which permits use, sharing, adaptation, distribution and reproduction in any medium or format, as long as you give appropriate credit to the original author(s) and the source, provide a link to the Creative Commons licence, and indicate if changes were made. The images or other third party material in this article are included in the article's Creative Commons licence, unless indicated otherwise in a credit line to the material. If material is not included in the article's Creative Commons licence and your intended use is not permitted by statutory regulation or exceeds the permitted use, you will need to obtain permission directly from the copyright holder. To view a copy of this licence, visit http://creativecommons.org/licenses/by/4.0/.

\section{References}

1. Zhu N, Zhang D, Wang W, Li X, Yang B, Song J, et al. A novel coronavirus from patients with pneumonia in China, 2019. N Engl J Med. 2020;382(8):727-33. https://doi.org/10.1056/ NEJMoa2001017.

2. Cao B, Wang Y, Wen D, Liu W, Wang J, Fan G, et al. A trial of lopinavir-ritonavir in adults hospitalized with severe covid-19. N Engl J Med. 2020. https://doi.org/10.1056/NEJMoa2001282.

3. Yao Y, Wang H, Liu Z. Expression of ACE2 in airways: implication for COVID-19 risk and disease management in patients with 
chronic inflammatory respiratory diseases. Clin Exp Allergy. 2020. https://doi.org/10.1111/cea.13746.

4. Djomkam ALZ, Olwal CO, Sala TB, Paemka L. Commentary: SARS-CoV-2 cell entry depends on ACE2 and TMPRSS2 and is blocked by a clinically proven protease inhibitor. Front Oncol. 2020;10:1448. https://doi.org/10.3389/fonc.2020.01448.

5. Shang J, Wan Y, Luo C, Ye G, Geng Q, Auerbach A, et al. Cell entry mechanisms of SARS-CoV-2. Proc Natl Acad Sci U S A. 2020;117(21):11727-34. https://doi.org/10.1073/pnas. 2003138117.

6. Huang C, Wang Y, Li X, Ren L, Zhao J, Hu Y, et al. Clinical features of patients infected with 2019 novel coronavirus in Wuhan, China. Lancet. 2020;395(10223):497-506. https://doi. org/10.1016/s0140-6736(20)30183-5.

7. Casanova JL, Su HC, Effort CHG. A global effort to define the human genetics of protective immunity to SARS-CoV-2 infection. Cell. 2020;181(6):1194-9. https://doi.org/10.1016/j.cell.2020.05. 016.

8. Hammarstrom L, Abolhassani H, Baldanti F, Marcotte H, PanHammarstrom Q. Development of passive immunity against SARS-CoV-2 for management of immunodeficient patients-a perspective. J Allergy Clin Immunol. 2020;146(1):58-60. https://doi. org/10.1016/j.jaci.2020.04.043.

9. Tangye SG, Al-Herz W, Bousfiha A, Chatila T, CunninghamRundles C, Etzioni A, et al. Human inborn errors of immunity: 2019 update on the classification from the International Union of Immunological Societies Expert Committee. J Clin Immunol. 2020;40(1):24-64. https://doi.org/10.1007/s10875-019-00737-x.

10. Abolhassani H, Kiaee F, Tavakol M, Chavoshzadeh Z, Mahdaviani SA, Momen T, et al. Fourth update on the Iranian National Registry of primary immunodeficiencies: integration of molecular diagnosis. J Clin Immunol. 2018;38(7):816-32. https://doi.org/10.1007/ s10875-018-0556-1.

11. Abolhassani H, Tavakol M, Chavoshzadeh Z, Mahdaviani SA, Momen T, Yazdani R, et al. National consensus on diagnosis and management guidelines for primary immunodeficiency. Immunology and Genetic Journal. 2019;2(1):1-21.

12. Seidel MG, Kindle G, Gathmann B, Quinti I, Buckland M, van Montfrans J, et al. The European Society for Immunodeficiencies (ESID) Registry Working Definitions for the Clinical Diagnosis of Inborn Errors of Immunity. J Allergy Clin Immunol Pract. 2019;7(6):1763-70. https://doi.org/10.1016/j.jaip.2019.02.004.

13. Abolhassani H, Hammarstrom L, Cunningham-Rundles C. Current genetic landscape in common variable immune deficiency. Blood. 2020;135(9):656-67. https://doi.org/10.1182/blood.2019000929.

14. Abolhassani H, Aghamohammadi A, Fang M, Rezaei N, Jiang C, Liu X, et al. Clinical implications of systematic phenotyping and exome sequencing in patients with primary antibody deficiency. Genet Med. 2019;21(1):243-51. https://doi.org/10.1038/s41436018-0012-x.

15. Itan Y, Shang L, Boisson B, Ciancanelli MJ, Markle JG, MartinezBarricarte R, et al. The mutation significance cutoff: gene-level thresholds for variant predictions. Nat Methods. 2016;13(2):10910. https://doi.org/10.1038/nmeth.3739.

16. Li Q, Wang K. InterVar: clinical interpretation of genetic variants by the 2015 ACMG-AMP guidelines. Am J Hum Genet. 2017;100(2):267-80. https://doi.org/10.1016/j.ajhg.2017.01.004.

17. Richards S, Aziz N, Bale S, Bick D, Das S, Gastier-Foster J, et al. Standards and guidelines for the interpretation of sequence variants: a joint consensus recommendation of the American College of Medical Genetics and Genomics and the Association for Molecular Pathology. Genet Med. 2015;17(5):405-24. https://doi. org/10.1038/gim.2015.30.

18. Härter G, Spinner CD, Roider J, Bickel M, Krznaric I, Grunwald S, et al. COVID-19 in people living with human immunodeficiency virus: a case series of 33 patients. Infection. 2020:1-6. https://oi. org/10.1007/s15010-020-01438-z.

19. Blanco JL, Ambrosioni J, Garcia F, Martínez E, Soriano A, Mallolas J, et al. COVID-19 in patients with HIV: clinical case series. Lancet HIV. 2020;7(5):e314-e6. https://doi.org/10.1016/ s2352-3018(20)30111-9.

20. Dinkelbach L, Franzel J, Berghauser MA, Hoehn T, Ghosh S, Lee U, et al. COVID-19 in a child with pre-existing immunodeficiency, cardiomyopathy, and chronic pulmonary disease. Klin Padiatr. 2020. https://doi.org/10.1055/a-1210-2639.

21. Soresina A, Moratto D, Chiarini M, Paolillo C, Baresi G, Focà E et al. Two X-linked agammaglobulinemia patients develop pneumonia as COVID-19 manifestation but recover. 2020.

22. Goetz L, Yang J, Greene W, Zhu Y. A COVID-19 patient with repeatedly undetectable SARS-CoV-2 antibodies. J Appl Lab Med. 2020. https://doi.org/10.1093/jalm/jfaa137.

23. Glowacka P, Rudnicka L, Warszawik-Hendzel O, Sikora M, Goldust M, Gajda P, et al. The antiviral properties of cyclosporine. Focus on coronavirus, hepatitis $\mathrm{C}$ virus, influenza virus, and human immunodeficiency virus infections. Biology (Basel). 2020;9(8). https://doi.org/10.3390/biology9080192.

24. Guo W, Ming F, Feng Y, Zhang Q, Mo P, Liu L, et al. Patterns of HIV and SARS-CoV-2 co-infection in Wuhan, China. J Int AIDS Soc. 2020;23(7):e25568. https://doi.org/10.1002/jia2.25568.

25. Quinti I, Lougaris V, Milito C, Cinetto F, Pecoraro A, Mezzaroma I, et al. A possible role for B cells in COVID-19? Lesson from patients with agammaglobulinemia. J Allergy Clin Immunol. 2020;146(1):211-3.e4. https://doi.org/10.1016/j.jaci.2020.04.013.

26. Gotzinger F, Santiago-Garcia B, Noguera-Julian A, Lanaspa M, Lancella L, Calo Carducci FI, et al. COVID-19 in children and adolescents in Europe: a multinational, multicentre cohort study. Lancet Child Adolesc Health. 2020. https://doi.org/10.1016/ S2352-4642(20)30177-2.

27. Parri N, Lenge M, Buonsenso D. Coronavirus infection in pediatric emergency departments research G. children with Covid-19 in pediatric emergency departments in Italy. N Engl J Med. 2020;383(2): 187-90. https://doi.org/10.1056/NEJMc2007617.

28. Chen G, Wu D, Guo W, Cao Y, Huang D, Wang H, et al. Clinical and immunological features of severe and moderate coronavirus disease 2019. J Clin Invest. 2020;130(5):2620-9. https://doi.org/ 10.1172/JCI137244.

29. Wang F, Nie J, Wang H, Zhao Q, Xiong Y, Deng L, et al. Characteristics of peripheral lymphocyte subset alteration in COVID-19 pneumonia. J Infect Dis. 2020;221(11):1762-9. https://doi.org/10.1093/infdis/jiaa150.

30. Diao B, Wang C, Tan Y, Chen X, Liu Y, Ning L, et al. Reduction and functional exhaustion of $\mathrm{T}$ cells in patients with coronavirus disease 2019 (COVID-19). Front Immunol. 2020;11:827. https:// doi.org/10.3389/fimmu.2020.00827.

31. Altmann DM, Boyton RJ. SARS-CoV-2 T cell immunity: specificity, function, durability, and role in protection. Sci Immunol. 2020;5(49). https://doi.org/10.1126/sciimmunol.abd6160.

32. Vabret N, Britton GJ, Gruber C, Hegde S, Kim J, Kuksin M, et al. Immunology of COVID-19: current state of the science. Immunity. 2020;52(6):910-41. https://doi.org/10.1016/j.immuni.2020.05. 002.

33. Meyts I, Bucciol G, Quinti I, Neven B, Fischer A, Seoane E, et al. Coronavirus Disease 2019 in patients with inborn errors of immunity: an international study. J Allergy Clin Immunol. 2020. https:// doi.org/10.1016/j.jaci.2020.09.010.

34. Mansourabadi AH, Sadeghalvad M, Mohammadi-Motlagh HR, Rezaei N. The immune system as a target for therapy of SARSCoV-2: a systematic review of the current immunotherapies for COVID-19. Life Sci. 2020;258:118185. https://doi.org/10.1016/j. lfs.2020.118185. 
35. Moraleda C, Serna-Pascual M, Soriano-Arandes A, Simo S, Epalza $\mathrm{C}$, Santos M, et al. Multi-Inflammatory syndrome in children related to SARS-CoV-2 in Spain. Clin Infect Dis. 2020. https://doi.org/ 10.1093/cid/ciaa1042.

36. Mehta P, McAuley DF, Brown M, Sanchez E, Tattersall RS, Manson JJ, et al. COVID-19: consider cytokine storm syndromes and immunosuppression. Lancet. 2020;395(10229):1033-4. https://doi.org/10.1016/S0140-6736(20)30628-0.

37. Huet T, Beaussier H, Voisin O, Jouveshomme S, Dauriat G, Lazareth I et al. Anakinra for severe forms of COVID-19: a cohort study. 2020.

38. van der Made CI, Simons A, Schuurs-Hoeijmakers J, van den Heuvel G, Mantere T, Kersten S, et al. Presence of genetic variants among young men with severe COVID-19. JAMA. 2020. https:// doi.org/10.1001/jama.2020.13719.

39. Zhang Q, Bastard P, Liu Z, Le Pen J, Moncada-Velez M, Chen J, et al. Inborn errors of type I IFN immunity in patients with lifethreatening COVID-19. Science. 2020. https://doi.org/10.1126/ science.abd4570.
40. Klocperk A, Parackova Z, Dissou J, Malcova H, Pavlicek P, Vymazal T, et al. Case Report: Systemic Inflammatory Response and Fast Recovery in a Pediatric Patient With COVID-19. Front Immunol. 2020;11:1665. https://doi.org/10.3389/fimmu.2020. 01665.

41. Castano-Jaramillo LM, Yamazaki-Nakashimada MA, Scheffler Mendoza SC, Bustamante-Ogando JC, Espinosa-Padilla SE, Lugo Reyes SO. A male infant with COVID-19 in the context of ARPC1B deficiency. Pediatr Allergy Immunol. 2020. https://doi. org/10.1111/pai.13322.

42. Bunyavanich S, Do A, Vicencio A. Nasal gene expression of angiotensin-converting enzyme 2 in children and adults. JAMA. 2020. https://doi.org/10.1001/jama.2020.8707.

43. Ludvigsson JF. Children are unlikely to be the main drivers of the COVID-19 pandemic - a systematic review. Acta Paediatr. 2020;109(8):1525-30. https://doi.org/10.1111/apa.15371.

Publisher's Note Springer Nature remains neutral with regard to jurisdictional claims in published maps and institutional affiliations.

\section{Affiliations}

\section{Samaneh Delavari ${ }^{1} \cdot$ Hassan Abolhassani $^{1,2}$ (D) Farhad Abolnezhadian ${ }^{3}$ - Fateme Babaha ${ }^{1,4} \cdot$ Sara Iranparast $^{5}$. Hamid Ahanchian ${ }^{6}$ - Nasrin Moazzen ${ }^{6}$. Mohammad Nabavi ${ }^{7}$. Saba Arshi ${ }^{7}$. Morteza Fallahpour ${ }^{7}$. Mohammad Hassan Bemanian ${ }^{7} \cdot$ Sima Shokri $^{7}$ - Tooba Momen ${ }^{8}$ - Mahnaz Sadeghi-Shabestari ${ }^{9} \cdot$ Rasol Molatefi $^{10}$. Afshin Shirkani ${ }^{11}$ - Ahmad Vosughimotlagh ${ }^{12} \cdot$ Molood Safarirad $^{12} \cdot$ Meisam Sharifzadeh $^{13} \cdot$ Salar Pashangzadeh $^{1}$. Fereshte Salami ${ }^{1}$ - Paniz Shirmast ${ }^{1}$ - Arezou Rezaei ${ }^{1}$ • Tannaz Moeini Shad $^{1}$ • Minoo Mohraz ${ }^{14}$. Nima Rezaei ${ }^{1}$. Lennart Hammarström ${ }^{2}$ - Reza Yazdani ${ }^{1}$ • Asghar Aghamohamamdi ${ }^{1}$}

1 Research Center for Primary Immunodeficiencies, Pediatrics Center of Excellence, Children's Medical Center, Tehran University of Medical Sciences, Tehran, Iran

2 Division of Clinical Immunology, Department of Laboratory Medicine, Karolinska Institute at Karolinska University Hospital Huddinge, Stockholm, Sweden

3 Department of Pediatrics, Abuzar Children's Hospital, Ahvaz Jundishapur University of Medical Sciences, Ahvaz, Iran

4 Department of Immunology, Faculty of Medical Sciences, Tarbiat Modares University, Tehran, Iran

5 Department of immunology, Faculty of Medical Sciences, Ahvaz Jundishapur University of Medical Sciences, Ahvaz, Iran

6 Allergy Research Center, Mashhad University of Medical Sciences, Mashhad, Iran

7 Department of Allergy and Clinical Immunology, Rasool e Akram Hospital, Iran University of Medical Sciences, Tehran, Iran

8 Department of Allergy and Clinical Immunology, Child Growth and Development Research Center, Research Institute for Primordial
Prevention of Noncommunicable Disease, Isfahan University of Medical Sciences, Isfahan, Iran

9 Immunology research center of Tabriz, TB and lung research center of Tabriz, children hospital, Tabriz University of Medical Science, Tabriz, Iran

10 Department of Pediatrics, Bo-Ali Children's Hospital of Ardabil University of Medical Sciences, Ardabil, Iran

11 Allergy and Clinical Immunology Department, Bushehr University of Medical Sciences, School of Medicine, Bushehr, Iran

12 Department of Pediatrics, North Khorasan University of Medical Sciences, Bojnurd, Iran

13 Division of Pediatric Critical Care, Children Medical Center, Tehran University of Medical Science, Tehran, Iran

14 Iranian Research Center for HIV/AIDS, Iranian Institute for Reduction of High-Risk Behaviors, Tehran University of Medical Sciences, Tehran, Iran 